\title{
TOWARDS A BETTER UNDERSTANDING OF ACTIVE GALACTIC NUCLEI ${ }^{1}$
}

\author{
PAOLO PADOVANI \\ Dipartimento di Fisica, II Università di Roma "Tor Vergata" \\ Via della Ricerca Scientifica 1, I-00133 Roma, Italy \\ E-mail: padovani@roma2.infn.it
}

\begin{abstract}
Active Galactic Nuclei (AGN) are ideal sources for multi-wavelength studies as their emission can cover almost 20 orders of magnitude in frequency from the radio to the $\gamma$-ray band. After reviewing their basic properties, I will assess how well we know the multifrequency spectra of AGN as a class. I will then briefly illustrate how currently available and forthcoming sky surveys will help in addressing some of the open questions of AGN studies. Finally, an analysis of the problem of the missing Type 2 QSO will exemplify the dangers of monochromatic sky surveys for AGN.
\end{abstract}

\section{AGN and Unified Schemes}

The large number of classes and subclasses which appear in AGN literature might disorientate astronomers working in other fields. A simplified classification, however, can be made based on radio loudness and the width of the emission lines (e.g., [18]). Radio-loud (RL) sources, that is objects with radio to optical ( $B$-band) flux ratio $f_{\mathrm{r}} / f_{\mathrm{B}} \gtrsim 10$ (e.g., [16]), make up only $\sim 10-15 \%$ of AGN and have, for the same optical luminosity, radio powers 3 to 4 orders of magnitude larger than those typical of radio-quiet (RQ) sources. AGN are also divided in Type 1 (broad-lined) and Type 2 (narrow-lined) objects according to their line-widths, with $1000 \mathrm{~km} / \mathrm{s}$ (full width half maximum) being the dividing value. We then have RL Type 1 AGN, that is radio quasars and broad-line radio galaxies, and RL Type 2 AGN, that is radio galaxies, with their corresponding RQ counterparts, that is Seyfert 1 galaxies and QSO, and Seyfert 2 galaxies respectively.

\footnotetext{
${ }^{1}$ Invited Review, to appear in New Horizons from Multi-Wavelength Sky Surveys, IAU Symposium 179, McLean B. et al. (eds.)
} 
Some objects exist with unusual emission line properties, such as BL Lacs, which have very weak emission lines with typical equivalent widths $<5 \AA$.

In recent years we have come to understand that classes of apparently different AGN might actually be intrinsically similar, only seen at different angles with respect to the line of sight. The basic idea, based on a variety of observations and summarized in Figure 1 of [18], is that emission in the inner parts of AGN is highly anisotropic. The current paradigm for AGN includes a central engine, surrounded by an accretion disk and by fast-moving clouds, probably under the influence of the strong gravitational field, emitting Doppler-broadened lines. More distant clouds emit narrower lines. Absorbing material in some flattened configuration (usually idealized as a torus) obscures the central parts, so that for transverse lines of sight only the narrow-line emitting clouds are seen (Type 2 AGN), whereas the near-IR to soft-X-ray nuclear continuum and broad-lines are visible only when viewed face-on (Type 1 AGN). In RL objects we have the additional presence of a relativistic jet, roughly perpendicular to the disk, which produces strong anisotropy and amplification of the continuum emission ("relativistic beaming"). In general, different components are dominant at different wavelengths. Namely, the jet dominates at radio and $\gamma$-ray frequencies (although it does contribute to the emission in other bands as well), the accretion disk is thought to be a strong optical/UV/soft X-ray emitter, while the absorbing material will emit predominantly in the IR.

This axisymmetric model of AGN implies widely different observational properties (and therefore classifications) at different aspect angles. Hence the need for "Unified Schemes" which look at intrinsic, isotropic properties, to unify fundamentally identical (but apparently different) classes of AGN. Seyfert 2 galaxies have been "unified" with Seyfert 1 galaxies, whilst lowluminosity and high-luminosity radio galaxies have been unified with BL Lacs and radio quasars respectively (see [2] and [18] and references therein).

\section{The multiwavelength spectrum of AGN}

The property that makes AGN ideal sources for multiwavelength studies is their broad-band emission, which covers basically the whole observable electromagnetic spectrum from the radio to the $\gamma$-ray band (almost 20 orders of magnitude in frequency). Multifrequency coverage at this level, however, is rare for a single object: see for example [1] and [9] which give simultaneous multifrequency data for NGC 3783, a Seyfert 1 galaxy, and 3C 273, a radio-loud quasar, respectively. Also, the objects studied are not necessarily representative of their class and are usually relatively local.

Single-object studies are certainly important, as specific models can be fitted to their multifrequency spectra to constrain the emission processes. 


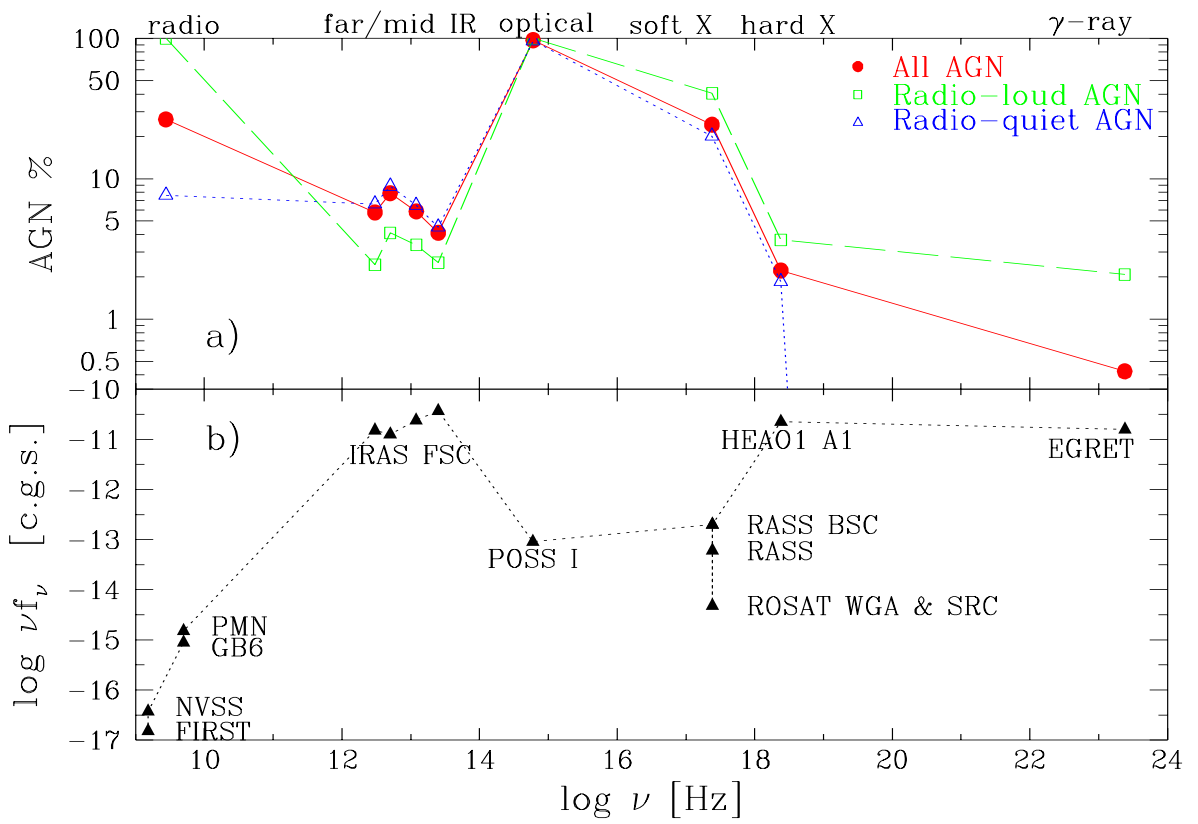

Figure 1. a) (top) The percentage of AGN with detections in a given band vs. frequency for all objects (solid points), radio-loud (open squares), and radio-quiet (open triangles) AGN. Sources without radio data are included in the latter class. b) (bottom) The sensitivity of the deepest all-sky (or very large area) surveys as a function of frequency.

However, one would also like to learn about the general properties of the AGN population and study statistically their emission in various bands, for example to constrain unified schemes. In other words, use multifrequency data to address some of the open questions of AGN research.

Before I address this point, we first have to assess how well we know the multifrequency spectrum of AGN as a class. I have then taken the latest Véron-Cetty \& Véron AGN catalog ([19]), which includes 11,662 AGN and gives redshift, optical (U, B, and $\mathrm{V}$ ) magnitudes, and some radio information (6 and $11 \mathrm{~cm}$ fluxes), and cross-correlated it with radio, far $/$ mid-IR, Xray and $\gamma$-ray catalogs available in machine readable form (using BROWSE as implemented at SAX/SDC). The list includes the FIRST and NVSS (respectively about $15 \%$ and $40 \%$ completed), the NORTH $20 \mathrm{~cm}, \mathrm{PKS}, \mathrm{PMN}$, GB6, S4, S5, 1 Jy catalogs (radio: 20, 11, and $6 \mathrm{~cm}$ ), the IRAS PSC and FSC (infrared: $12-100 \mu$ ), the EXOSAT CMA, the ROSAT RASS-BSC and WGA, the Einstein Slew, EMSS, IPC catalogs (soft X-ray: $~ 0.05-3.5$ $\mathrm{keV}$ ), a hard X-ray compilation ([10]), and the GRO EGRET catalog ( $\gamma$ ray: $30 \mathrm{MeV}-20 \mathrm{GeV}$ ). Many of these surveys have been discussed at this meeting by various contributions, where detailed references can be found. 
Figure 1a, which summarizes more than 30 years of observations, shows the percentage of AGN with data in a given band as a function of frequency for all AGN and for the RL and RQ subclasses. It can be seen that the multifrequency spectrum of AGN is not that well known. We are not doing too badly in the radio (at a few $\mathrm{GHz}$ ) and soft X-rays, where about $30 \%$ of AGN are detected, while basically all AGN have optical data (due to the fact that the eye is peaked in the optical and we always require an "optical identification"). However, we have major "holes" in our knowledge in the far/mid-IR, hard X-ray, and $\gamma$-ray bands. Note also that RL AGN fare better than RQ ones at all frequencies, apart from the IRAS band. If we restrict ourselves to the radio/optical/soft X-ray bands, where we have the best coverage, only about $10 \%$ of AGN have data in all three bands.

The fractions of objects shown in Fig. 1a are the convolution of two effects: 1 . the intrinsic spectral shape of AGN; 2 . the sensitivity of our detectors in the various bands. The latter is shown in Fig. 1b, which displays the limiting sensitivities of the deepest all-sky (or large-area) surveys available at various frequencies, which were used to construct Fig. 1a (deeper, smallarea surveys exist in some bands). The POSS I limit, as discussed above, is only indicative as many optical data come from dedicated observations. The main point here is that the bands where we have less information, i.e. far/mid-IR, hard X-ray and $\gamma$-ray, are exactly those where our surveys are less sensitive. It then follows that our poor knowledge of the multifrequency spectra of AGN is due, more than to their intrinsic faintness in some bands, to the limitations of our detectors. (The radio band is an exception: radio surveys are very deep but most AGN are weak radio sources.)

\section{The role of multiwavelength sky surveys in AGN research}

Given the broad-band emission of AGN, it is clear that multiwavelength

sky surveys are extremely important to further our understanding of AGN physics, at a zero order simply by providing more data for more AGN.

This is not as trivial as it might sound: AGN are rare, making up only about $1 \%$ of all bright galaxies (although low luminosity AGN [e.g., LINERs] could be relatively more numerous). The latest AGN catalog includes about 12,000 sources, while at this meeting catalogs with tens and even hundreds of millions of entries have been discussed (e.g., [5]). AGN are also hard to find, especially in the optical. Some of the complete samples, which are needed to study AGN evolution, are still quite small, especially the radio-selected ones, and only less than 300 BL Lacs are known over the whole sky ([13]). The cross-correlation between surveys will take advantage of the broad-band emission of AGN, enhancing the efficiency of AGN detection, especially important for rare classes of objects ([11] and [14]). 
Specific examples of open questions in AGN research which will benefit from existing and future sky surveys include the following:

- The radio-loud/radio-quiet dichotomy. Despite years of effort, we still do not know what makes an AGN radio-loud nor why the $f_{\mathrm{r}} / f_{\mathrm{B}}$ distribution is bimodal for optically-selected samples. Deep radio surveys, like the NVSS and the FIRST, will allow us to study for the first time large numbers of radio-selected radio-quiet objects ([7]), to check if the $f_{\mathrm{r}} / f_{\mathrm{B}}$ distribution is still bimodal when the selection is done in the radio band, and even to study the radio evolution of RQ quasars.

- Thermal versus non-thermal emission. While we have strong evidence in favour of a dominance of non-thermal (synchrotron and inverse Compton) emission in radio-loud AGN, the situation is not that clear for radio-quiet sources, although the optical/UV might be dominated by thermal emission. Multifrequency data for large numbers of AGN are needed to address this question on a statistical basis.

- AGN Evolution. The driving force behind the strong evolution observed in AGN is still not understood. Also, although the form of the evolution seems to be similar at radio, optical, and X-ray frequencies, this should be checked against larger and deeper samples.

This list is no doubt incomplete but it is only meant to give a flavor of the possibilities multiwavelength surveys provide us with. I will now concentrate on a particular problem, which is a text-book example of how monochromatic surveys can be misleading: the missing Type 2 QSO.

\section{The mystery of the missing Type 2 QSO}

According to unified schemes, Type 2 objects are Type 1's seen edge-on, i.e., with their central parts strongly obscured by dust. Seyfert 2's have been identified as the Type 2 equivalent of Seyfert 1's, and radio galaxies as the Type 2 equivalent of radio quasars. We also think that Seyfert 1 galaxies are simply low-luminosity, low-redshift versions of RQ quasars. There should then be Type 2, i.e., narrow-lined, RQ quasars: but where are they?

Figure 2 shows the redshift distribution of all RQ Type 1 and Type 2 AGN in [19]. Most known Type 2's are local, $\sim 80 \%$ of them at $z<0.1$, and there seem to be no high- $z$ counterparts to the local Seyfert 2's. Note that for semi-opening angles of the obscuring torus between $30^{\circ}$ and $45^{\circ}$ (derived from various methods), Type 2 AGN should be intrinsically more numerous than Type 1's by factors between 6.5 and 2.4, that is most RQ AGN should be of Type 2. Even for angles as large as $60^{\circ}$ the two classes should be equally numerous. The point is that most quasars are still identified by optical searches, tuned to find AGN with strong non-stellar continua, i.e. Type 1 quasars. Normally, in fact, candidates are first selected on the basis of 


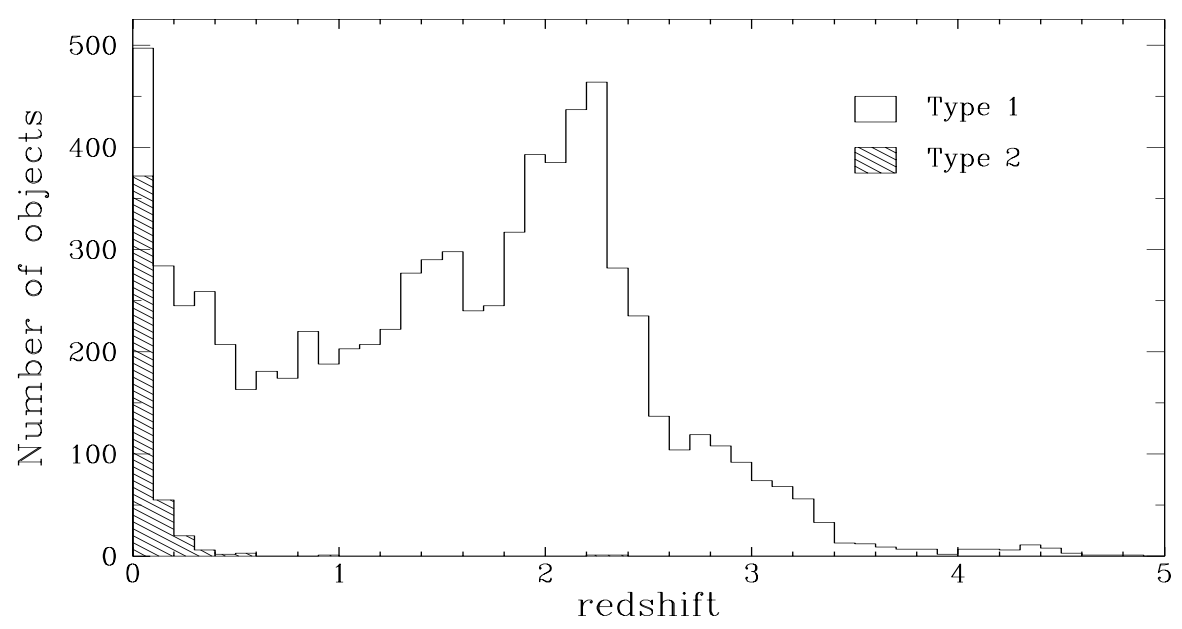

Figure 2. The redshift distribution of the $\sim 8,000$ radio-quiet AGN in the Véron-Cetty \& Véron catalog for Type 1 (broad-lined) and Type 2 (narrow-lined) objects. Only 3 Type 2 radio-quiet AGN are known at $z>0.9$.

their non-stellar colors, then observed spectroscopically to see if they show broad lines. The inferred extinction in Type 2 objects can be larger than 10 magnitudes, reaching even values as high as a few hundred (e.g., [6]), so in these objects the AGN continuum is swamped by the host galaxy light. Type 2 QSO, then, do not even make it to the candidate level, and even if they did, they would be discarded because they do not show broad lines! Type 2 radio sources, of course, are selected because of their radio emission and, in fact, we have examples of radio galaxies at redshifts almost as high as those of radio quasars. Seyfert 2's, on the other hand, are relatively easy to find locally where the selection is based on host galaxy properties. For example, a complete sample of Seyferts can be derived by taking spectra of all galaxies down to a given magnitude limit. This is of course only feasible for relatively bright (and therefore local) galaxies (e.g., $B \leq 14.5$ : [8]).

How can we find the high-redshift Type 2 QSO? The energy absorbed by the obscuring material in the optical/UV/soft X-ray bands will have to be re-emitted in the IR band. Type 2 AGN should then be strong IR emitters. In fact, optical identification of IRAS sources has produced a large number of Type 2 AGN, approximately $30 \%$ of known objects, and IRAS selected Type 2's make up about $50 \%$ of the few sources at $z>0.1$. Some of these objects have also, as expected, bolometric luminosities typical of quasars. However, the redshift distribution in Fig. 2 includes all these IRAS selected Type 2 AGN, which leads to the next question: Why is IRAS not finding more of them? The problem is that the IRAS survey, as illustrated in Fig. 
$1 \mathrm{~b}$, is not very sensitive: IRAS has detected only about $8 \%$ of all known AGN at $60 \mu$ (and an even smaller fraction in the other bands) and these are mostly local sources, $\sim 80 \%$ at $z<0.1$. IRAS is then sampling only the local universe and the few high-z Type 2 AGN detected are probably atypically luminous, like IRAS F10214+4724 $(z=2.286)$, which has recently been shown to be gravitationally lensed ([4]).

The torus becomes transparent at hard X-ray energies but even the surveys in this band, made with the HEAO1 observatory, are not very sensitive: only about $2 \%$ of known AGN have hard X-ray data $(\sim 10 \mathrm{keV})$ and again most of them are local sources $(z<0.1)$.

\section{Towards an unbiased survey}

If our current understanding of AGN is correct, the example I have just given is not isolated. Most available surveys, in fact, give us a "biased" view of the AGN population, not only because different components emit at different frequencies, but mainly because most surveys preferentially detect some particular classes of AGN. Namely, high-frequency radio surveys are dominated by objects with beamed radio emission, the so-called blazars (BL Lacs and core-dominated quasars). Although this is less of a problem at the faint fluxes reached by the FIRST and NVSS surveys, it can be shown that even these surveys will not detect the bulk of the RQ population, so that radio surveys still preferentially detect RL AGN. (Low-frequency radio surveys are less biased in terms of selecting beamed objects but still detect mostly RL sources). The region between near-IR to soft X-ray frequencies is the most biased of all: here in fact the torus is optically thick and only the nuclear continuum emission of Type 1 AGN is detected. Radiation from the central parts manages to escape only at $\lambda \gtrsim 50-100 \mu$ and $E \gtrsim 10-20 \mathrm{keV}$, the precise values depending on the density and geometry of the absorbing column. (A likely distribution of these parameters also implies a distribution in the energies at which we can see through the torus.) The $\gamma$-ray EGRET sky survey is also biased, as it has only detected blazars. Far-IR surveys, as discussed above, are unbiased, but current surveys are not deep enough (also, they suffer from strong contamination by non-AGN sources, mostly star-forming regions, both galactic and extragalactic). Finally, hard X-ray surveys are also unbiased, but still not deep enough.

What about future far-IR and hard X-ray surveys? As discussed at this meeting ([3]), our best bet for a large area, far-IR survey is the COBRAS/ SAMBA mission, which will perform all-sky surveys in the $30-900 \mathrm{GHz}$ range. In fact, as regards other IR surveys, they will either be barely more sensitive than IRAS (e.g., the ISO $200 \mu$ serendipitous survey) or will only sample the mid-IR range (e.g., WIRE) or be limited to small areas of the 
sky (e.g., ISO ELAIS [12], SIRTF). As regards hard X-rays, there will be serendipitous surveys covering the $2-30 \mathrm{keV}$ (SAX WFC) and $50-100$ $\mathrm{keV}$ (GRO BATSE) ranges but as these instruments were made for other purposes they will not be much more sensitive than the HEAO1 surveys. On the other hand, the SAX LECS and MECS will provide a deep serendipitous survey in the $1-10 \mathrm{keV}$ band, while deep surveys will also be performed in the $0.25-12 \mathrm{keV}$ and $2-12 \mathrm{keV}$ ranges respectively by XMM ([15]) and ABRIXAS ([17]), the former in serendipitous and slew mode, the latter as a proper all-sky survey. Although these energy ranges are not optimized to see through the torus in all sources, the rest-frame frequency increases as $(1+\mathrm{z})$ so that at $z=1$, for example, ABRIXAS will sample the $4-24 \mathrm{keV}$ range. These surveys will certainly represent a major step forward in our quest to obtain an unbiased view of AGN, independent of orientation.

In summary, the main conclusions are as follows: 1 . both because different AGN components emit in different bands and because most sky surveys are biased towards particular AGN classes, no single survey can give us the broad view of AGN we need to understand them. Therefore, multiwavelength sky surveys are vital for AGN research. 2. If our understanding of AGN is correct, we may be missing between $70 \%$ to $90 \%$ of (radio-quiet) AGN. Deep far-IR and hard X-ray surveys are needed to see through the absorbing material we think surrounds the central parts of AGN and identify the large numbers of AGN which have so far escaped detection.

Acknowledgements. Many thanks to various participants in the IAU 179 Symposium for useful discussions. I also acknowledge helpful comments from two long-standing collaborators, Meg Urry and Paolo Giommi, the latter being also responsible for introducing me to the world of database management.

\section{References}

1. Alloin, D., et al., 1995, A\&A, 293, 293

2. Antonucci, R., 1993, ARAA, 31, 473

3. Beichman, C., these proceedings

4. Broadhurst, T. and Lehar, J., 1995, ApJ, 450, L41

5. Canzian, B., these proceedings

6. Goodrich, R.W., Veilleux, S. and Hill, G.J., 1994, ApJ, 422, 521

7. Gregg, M.D., et al., 1996, AJ, 112, 407

8. Hucra, J. and Burg, R., 1992, ApJ, 393, 90

9. Lichti, G.G., et al., 1995, A\&A, 298, 711

10. Malizia, A. and Bassani, L., 1996, private communication

11. Nass, P., et al., these proceedings

12. Oliver, S., et al., these proceedings

13. Padovani, P. and Giommi, P., 1995, MNRAS, 277, 1477

14. Perlman, E., et al., these proceedings

15. Stewart, G., these proceedings

16. Stocke, J.T., Morris, S.L., Weymann, R.J. and Foltz, C.B., 1992, ApJ, 396, 487 
17. Trümper, J., these proceedings

18. Urry, C.M. and Padovani, P., 1995, PASP, 107, 803

19. Véron-Cetty, M.-P., and Véron, P., 1996, ESO Scientific Report No. 17 